\title{
Computer Navigation in Minimally Invasive Spine Surgery
}

\author{
Jonathan N. Sembrano ${ }^{1}$. Sharon C. Yson ${ }^{1}$ - Jeffrey J. Theismann ${ }^{1}$
}

Published online: 7 November 2019

(C) Springer Science+Business Media, LLC, part of Springer Nature 2019

\begin{abstract}
Purpose of Review The goal of the review is to discuss the common general applications of navigation in the context of minimally invasive spine surgery and assess its value in the published literature comparing against non-navigated or navigated techniques.

Recent Findings There is increasing utilization of computer navigation in minimally invasive spine surgery. There is synergy between navigation and minimally invasive technologies, such that one enhances or facilitates the other, thus leading to wider applications for both. Specifically, navigation has been shown to improve performance of percutaneous pedicle screw placement, vertebral augmentation, and minimally invasive fusion procedures. Overall, clinical studies have shown better accuracy and less radiation exposure with the use of navigation in spine surgery.

Summary The use of navigation in minimally invasive spine surgery enhances the accuracy of instrumentation and decreases radiation exposure. It is yet to be determined whether patient-reported outcomes will differ. Further research on its effect on clinical outcomes may further define the future impact of navigation in minimally invasive spine surgery.
\end{abstract}

Keywords Navigation $\cdot$ Minimally invasive spine surgery $\cdot$ Spine fusion $\cdot$ Percutaneous pedicle screw

\section{Introduction}

Computer navigation has allowed for more accurate and thus safer placement of spinal instrumentation. Although navigation was initially used mainly in conventional open approach spine surgery, proliferation and increasing utilization of minimally invasive surgical techniques prompted the adoption of navigation in minimally invasive surgery (MIS) as well. It can also be considered that, in some ways, navigation has facilitated the development of MIS, as it has allowed surgeons to

This article is part of the Topical Collection on Minimally Invasive Spine Surgery

Jonathan N. Sembrano

sembr001@umn.edu

Sharon C. Yson

scyson@umn.edu

Jeffrey J. Theismann

theis312@umn.edu

1 Department of Orthopaedic Surgery, University of Minnesota Medical School, 2450 Riverside Ave S R200,

Minneapolis, MN 55454, USA overcome the main limitation of MIS surgery, which is that of limited direct visualization.

This article will discuss and review the literature on the common general applications of navigation technology in MIS spine surgery: percutaneous pedicle screw fixation, vertebral augmentation (i.e., vertebroplasty/kyphoplasty), minimally invasive transforaminal lumbar interbody fusion (MIS TLIF), and lateral/oblique lumbar interbody fusion (LLIF/OLIF).

\section{Navigated Percutaneous Pedicle Screw Fixation (Table 1)}

The most common application of computer navigation in spine surgery is in pedicle screw placement. Since surgeons began placing pedicle screws as spinal fixation anchors, many published studies have focused on screw placement accuracy, given the potential catastrophic consequences of screw malposition. There has been increasing emphasis on limiting approach-related morbidity with percutaneous screws being placed with minimizing muscle stripping and injury. However, with the advantages of minimal tissue damage, came at the cost of losing direct visualization of anatomic landmarks which was mitigated by the heavy use of 


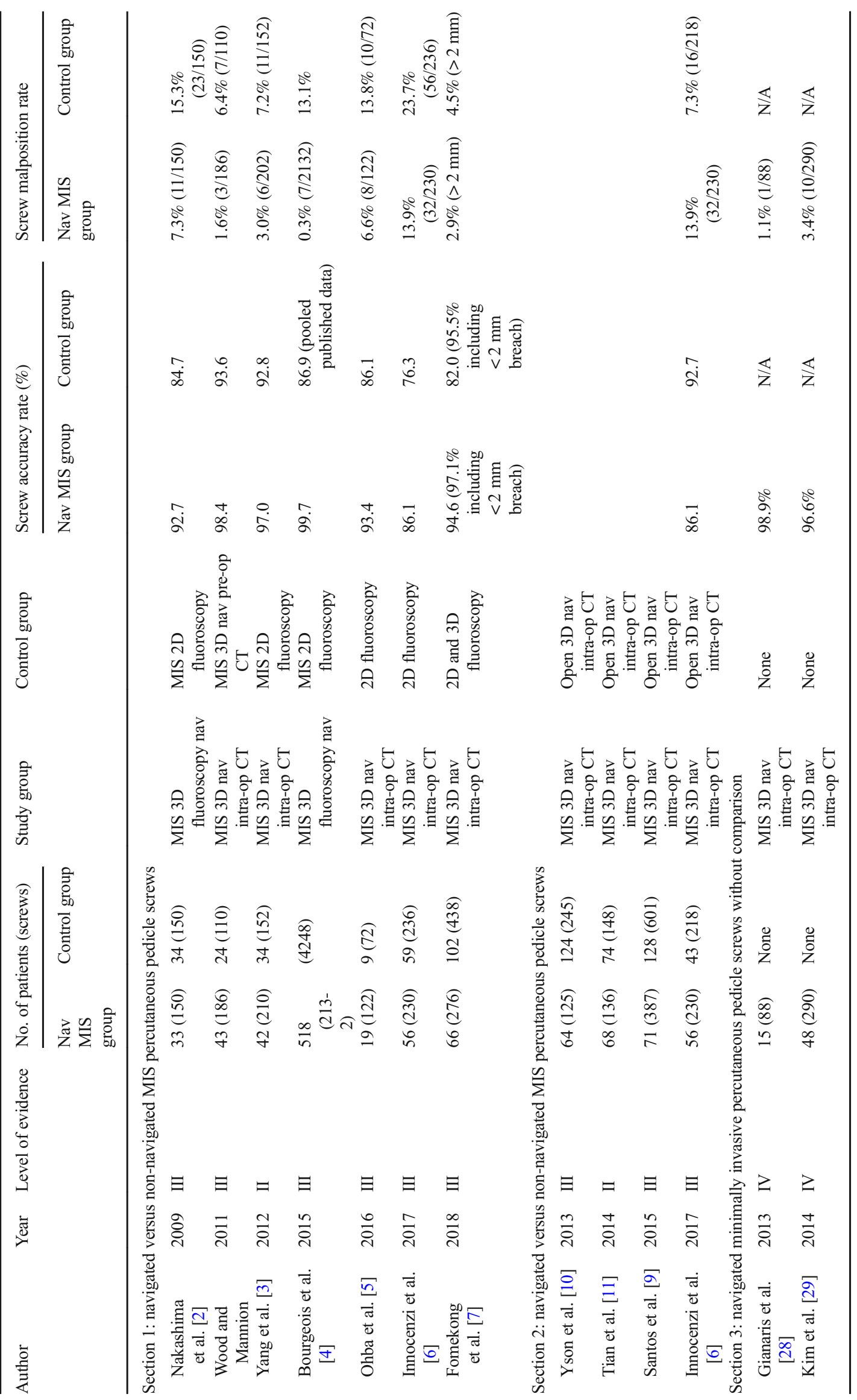




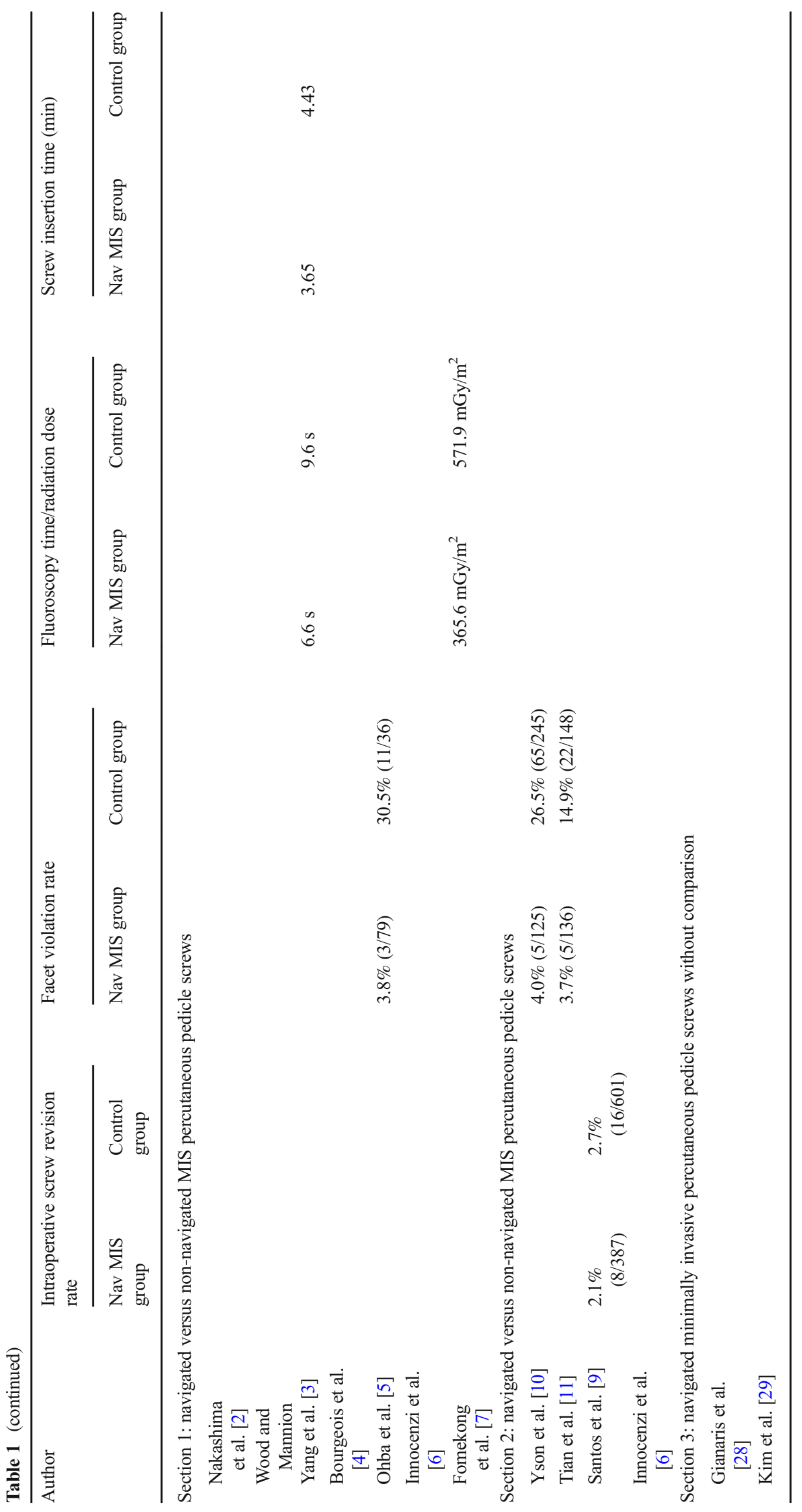


intraoperative fluoroscopy. Unfortunately, this brought about significantly increased radiation exposure, not only to the patient but more so to the surgical team, with repetitive use of fluoroscopy. Many surgeons who are hesitant to adopt MIS techniques in their practice cite increased radiation exposure as one of the reasons for not doing so [1].

With the use of computer navigation, surgeons could reap the benefits of minimally invasive surgery and, at the same time, improve screw placement accuracy and minimize radiation exposure. In 2009, Nakashima et al. [2] published a retrospective study comparing navigated MIS versus MIS screws placed using conventional fluoroscopy. They found that the navigated group had better accuracy than the fluoroscopy group $(92.7 \%$ vs. $84.7 \%)$. Other studies published since that also compared MIS navigation against MIS conventional fluoroscopy and likewise consistently found superior screw placement accuracy when computer navigation was utilized [3-7], with accuracy rates ranging from $86.1 \%$ [6] to $99.7 \%$ [4].

Wood and Mannion [8] compared two navigation techniques: one utilizing intraoperatively acquired CT images versus an older method utilizing preoperative $\mathrm{CT}$ scans requiring intraoperative registration. They found that the newer method using intraoperative CT yielded more accurate screw placement $(98.4 \%$ vs. $93.6 \%, P$ value $=0.03)$.

Santos et al. [9] compared intraoperative screw revision rates between navigated MIS and navigated open screw placement and found that there was no significant difference in intraoperative revision rate $(2.1 \%$ vs. $2.7 \%)(P=0.0004)$. None of the patients needed a reoperation for screw revision in their series. Innocenzi et al. [6] published a series comparing 4 groups of pedicle screw placement, among which were navigated MIS and navigated open screw placement, which showed a higher screw malposition rate in the MIS group $(13.9 \%$ vs. $7.3 \%)(P=0.0263)$.

Two studies looked at facet joint violation rates $[10,11]$. Yson et al. [10], in a series of 188 patients (370 screws), found significantly lower cranial (top end of construct) facet violation rates with navigated percutaneous screws versus navigated open screws $(4.0 \%$ vs. $26.5 \%)(P<0.0001)$. The authors attributed this to enhanced ability to achieve a more medial trajectory and lateral screw entry point with the percutaneous technique compared to open screw placement. Similarly, Tian et al. [11], in a prospective comparative (level 2) study of 142 patients (284 screws), found lower facet violation rates in navigated percutaneous versus navigated open screws $(3.7 \%$ vs. $14.9 \%$ ).

Most studies have utilized an optical camera that is able to track passive reflective markers/spheres and required direct line of sight between the camera and the spheres. Images are obtained from either preoperative or intraoperative CT, and registration is either manual (point by point) or automated (where the camera is able to "see" the scanner by detecting active or passive markers on the scanner as well at the time of image acquisition). A cadaveric study by von Jako [12] in 2010 utilized electromagnetic field (EMF) navigation. They placed 122 screws in 8 cadavers, 62 screws placed under fluoroscopic guidance, and 60 screws under EMF guidance. They found EMF to have statistically significant improvements in the lumbar spine, both ideal trajectory $(62.7 \%$ vs. $40 \%, P=0.01)$ and rate of pedicle breaches $(16.2 \%$ vs. $42.5 \%, P=0.01$ ), but overall found a non-significant change in overall accuracy rate between the two techniques ( $83 \%$ vs. $71 \%, P=0.12$ ).

Overall, these studies show generally high accuracy rates when MIS/percutaneous screws are placed, utilizing navigation technology. Those studies that utilized a control group, either fluoroscopy guided or freehand using anatomic landmarks, consistently showed higher accuracy rates with navigation.

\section{Navigated Vertebral Augmentation (Vertebroplasty/Kyphoplasty) (Table 2)}

The indications for percutaneous cement augmentation for osteoporotic or pathologic/metastatic compression fractures are controversial, with studies supporting either that they provide significant pain relief for suffering patients or that they are no better compared to sham/placebo interventions. Augmentation may be performed either with (kyphoplasty) or without (vertebroplasty) the use of balloon inflation prior to cement injection. Several studies have looked at the use of navigation technology for cement augmentation procedures.

In 2005, Villavicencio et al. [13] published a small series comparing navigated kyphoplasty in 11 patients with nonnavigated kyphoplasty in 9 patients. There was no significant difference in operative time; however, they found a significant reduction in fluoroscopy time ( $81.3 \mathrm{~s}$ in the navigated group vs. $293.2 \mathrm{~s}$ in the non-navigated group) $(P=0.02)$. In their conclusion, the authors emphasized the advantage of significant reduction in radiation exposure with the use of navigation.

Izadpanah et al. [14] also looked at fluoroscopy time; additionally, they also measure radiation dose via dose area product (DAP) [14]. They found decreased fluoroscopy times in the navigation group $(99 \mathrm{~s}$ vs. $175 \mathrm{~s}$ for thoracic levels; $74 \mathrm{~s}$ vs. $165 \mathrm{~s}$ for lumbar levels). They also found decreased radiation dose in the navigation group $\left(1245 \mathrm{cGy} / \mathrm{cm}^{2}\right.$ vs. 1972 $\mathrm{cGy} / \mathrm{cm}^{2}$ for thoracic levels; $1318 \mathrm{cGy} / \mathrm{cm}^{2}$ vs. $2105 \mathrm{cGy} / \mathrm{cm}^{2}$ for lumbar levels).

Sun et al. [15] looked at needle positioning accuracy and cement extravasation rates between navigated and nonnavigated kyphoplasty cases. They found a lower rate of need for needle adjustment in the navigation group (5.3\% vs. $33 \%)$, significance not analyzed. Cement extravasation rates were, however, similar between the two groups (31\% navigation 


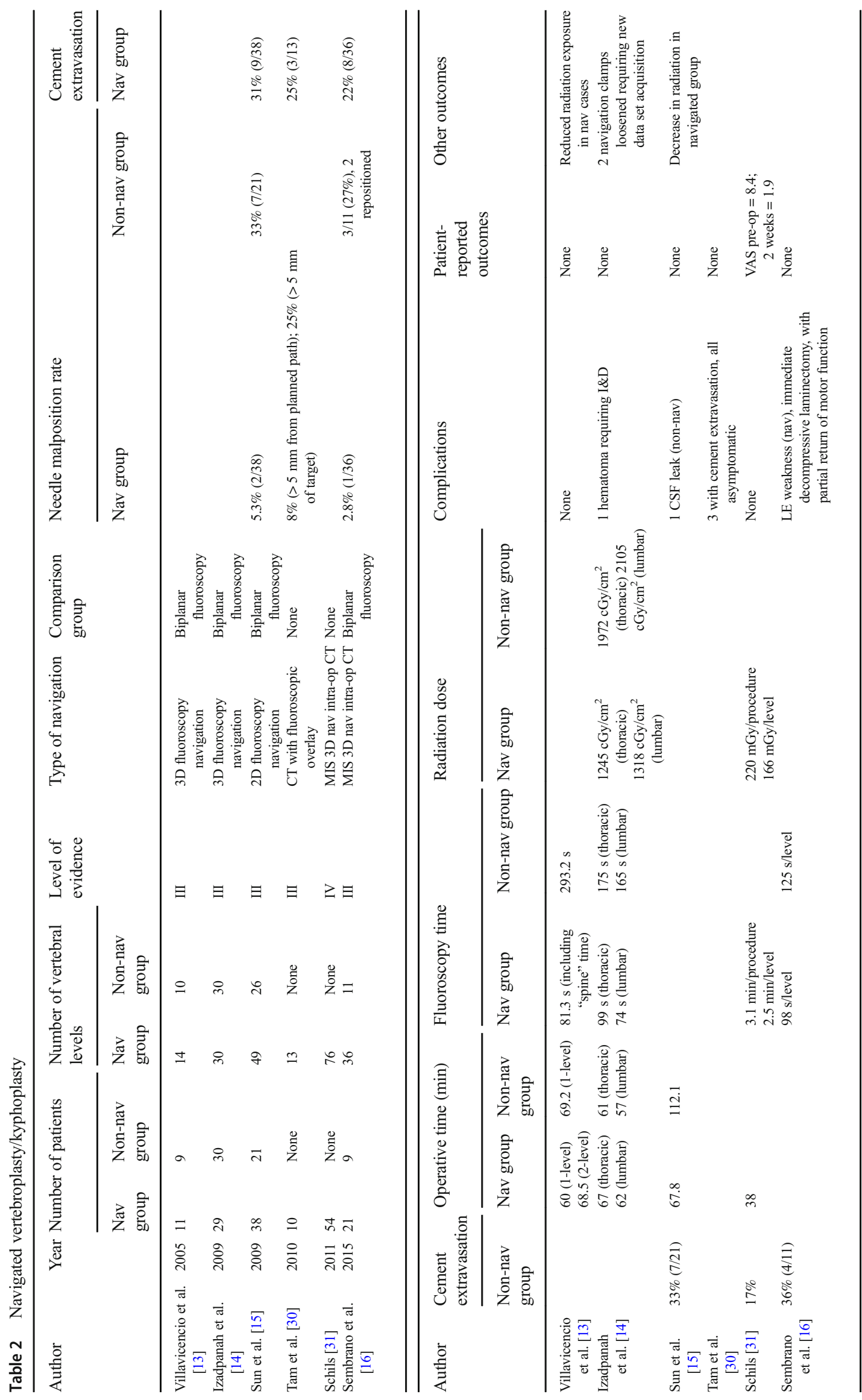


(nav), 33\% non-nav). Similarly, Sembrano et al. [16] compared navigated versus non-navigated kyphoplasty and found a much lower needle malposition rate in the navigation group ( $2.8 \%$ vs. $27 \%, P=0.04)$. However, there was again no significant difference in cement extravasation rates $(22 \%$ nav vs. $36 \%$ non-nav). The authors attributed this to the fact that needle positioning was directly dependent on the navigation, whereas cement is injected in a fluid state and follows paths of least resistance.

\section{Navigated MIS TLIF (Table 3)}

TLIF is perhaps the most commonly performed interbody fusion approach in the lumbar spine, mainly because of its versatility (i.e., could be performed at any level) and utilization of a single position and approach for decompression, access to the disc space, and screw placement. This procedure can be performed either via conventional open posterior approach or via muscle-splitting minimally invasive approach (MIS TLIF).

The first report on navigated MIS TLIF was by Kim et al. [17] in 2008. They compared 10 patients who had 2D fluorobased navigated MIS TLIF versus 8 patients who had nonnavigated surgery. In their description of the technique, navigation was used to select skin incision site, to dock the tubular retractor at the right angle in line with the target disc level, and to place the pedicle screws. They found $100 \%$ screw accuracy in both groups, but with significantly lower fluoroscopy time in the navigated group $(147.2 \mathrm{~s}$ vs. $57.1 \mathrm{~s}, P=0.02)$ Other published case series (level IV studies) using computer navigation for MIS TLIF included Luo et al. [18], Cho et al. [19], and Zhang et al. [20]. In these reports, it would seem that navigation was used primarily for percutaneous pedicle screw placement including planning of skin incision. After screw placement, the TLIF procedure itself usually proceeded with little use or need for navigation.

Two retrospective comparative studies have been published [21, 22]. Tian et al. [21] compared 30 3D imagebased navigated MIS TLIF cases against 31 non-navigated open TLIF cases. For the MIS TLIF group, navigation was used to plan skin incisions and to place screws. They found reduced OR time, blood loss, postoperative drain output, and pain in the navigated group. Xu et al. [22] reported on 74 consecutively navigated MIS TLIF cases and compared the first 25 with the latter 74 cases. They found decreased surgical and anesthesia time in the latter group attributable to the learning curve. They concluded that it takes $\sim 25$ cases to become proficient at navigated MIS TLIF.

\section{Navigated LLIF/OLIF (Table 4)}

LLIF, also commonly referred to as extreme/direct lumbar interbody fusion (XLIF or DLIF, respectively), and OLIF are minimally invasive approaches to lumbar interbody fusion performed with the patient in the lateral decubitus position. The basic difference is that LLIF is a transpsoas approach and OLIF passes anteriorly to the psoas. The use of intraoperative imaging is necessary because of the limited direct visualization inherent in minimally invasive surgery. It is thus a natural evolution of these procedures for surgeons to seek ways to mitigate radiation exposure by using navigation technology.

In 2015, Park [23] reported on a series of 8 patients on whom LLIF with intraoperative CT computer navigation was performed, and concluded that computer navigation for LLIF procedures is feasible and safe. In 2018, Jiang et al. [24] published a comparative retrospective study on 18 patients who underwent a similar navigated LLIF procedure versus 15 patients who underwent non-navigated surgery. They found no difference in operative time or fusion rates but found significantly lower fluoroscopy time in the navigated group ( $13.5 \mathrm{~s}$ vs. $53.2 \mathrm{~s})$. Notably, all the surgeries in their series were performed for spinal tuberculosis.

For the OLIF procedure, Zhang et al. [25] published in 2017 a retrospective study comparing 22 patients who underwent intraoperative CT navigated OLIF versus 20 patients who underwent conventional OLIF with 2D fluoroscopy. They also found significantly lower radiation exposure to both the patient (44.6 mGy vs. $9.4 \mathrm{mGy}$ ) and the surgeon (44.6 mGy vs. 0 mGy). Operative time was slightly longer in the navigated group $(2.5 \mathrm{~h}$ vs. $2.3 \mathrm{~h})$ but not statistically significant. The authors concluded that navigation is a safe alternative to fluoroscopy for OLIF, and that it can eliminate radiation exposure to the surgeon and decrease exposure to the patient. In the same year, DiGiorgio et al. published on a larger series of 49 patients who underwent similar navigated OLIF [26]. They reported improvement in back and leg pain scores postoperatively.

In 2018, Sellin et al. [27] reported on a small series of 4 patients who underwent simultaneous OLIF and percutaneous pedicle screw fixation using intraoperative CT-guided navigation in the lateral position. While the authors clearly described using navigation for screw placement, it was less clear whether and how it was used for the OLIF portion of the procedure as well.

\section{Conclusion}

Computer navigation technology, as applied to minimally invasive spine surgery, has been consistently shown to significantly enhance accuracy in placing spinal instrumentation, thus increasing safety for the patient, likely improving the biomechanical performance of the construct and reducing costly injuries and reoperations. It has also shown significant promise in reducing radiation exposure and dose and may help mitigate the harmful long-term effects of ionizing radiation. It 


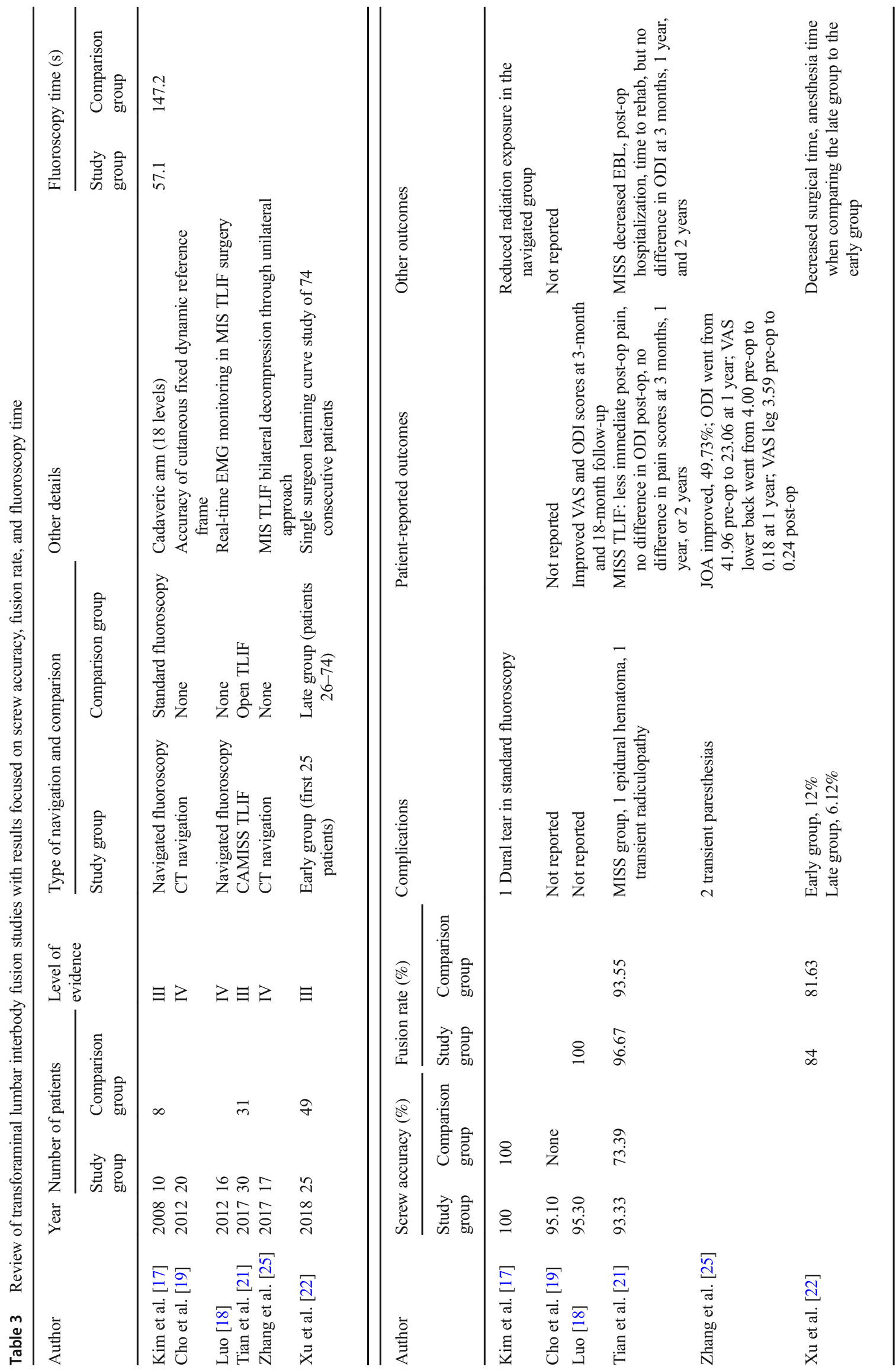




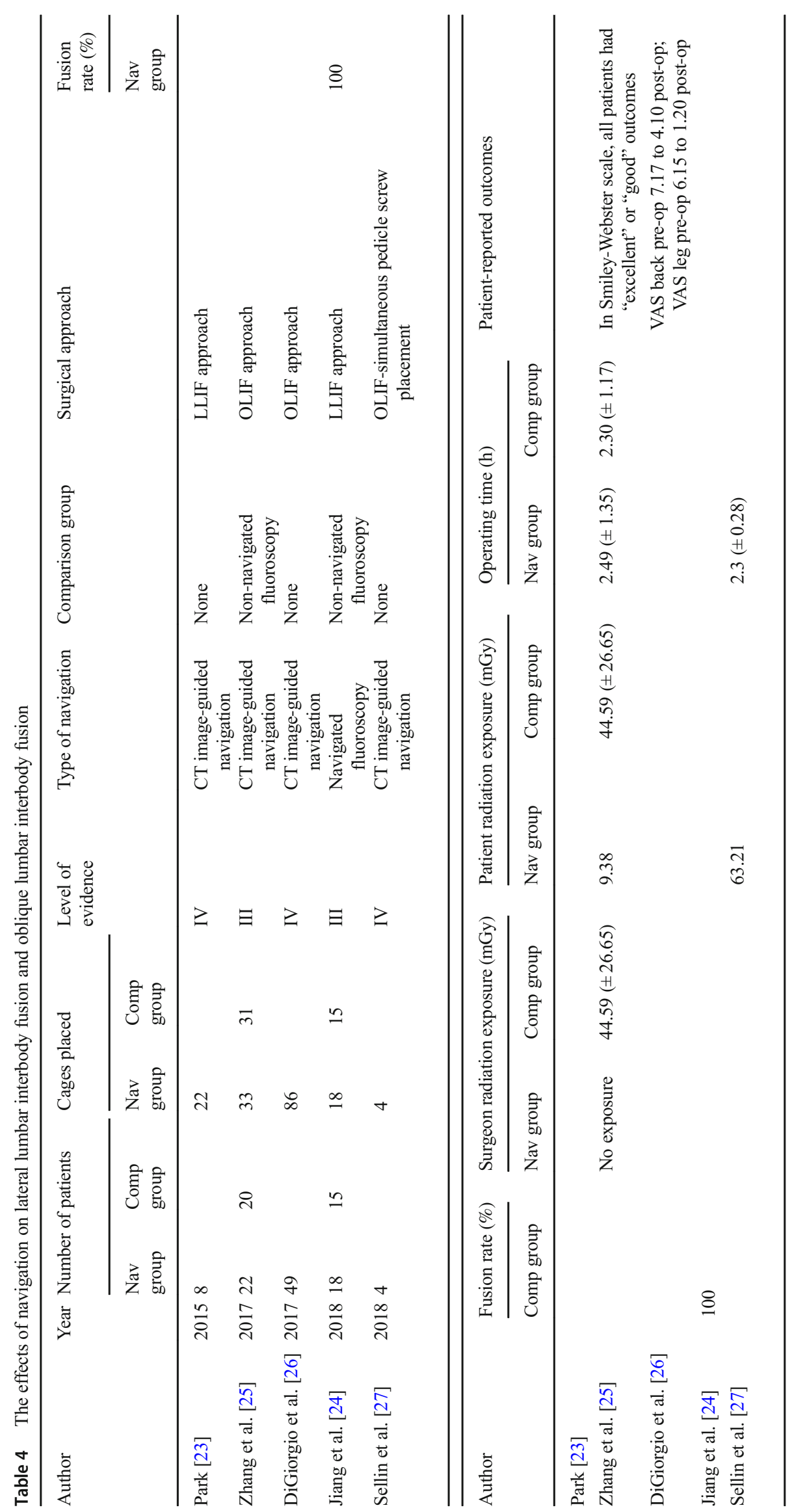


is thus highly advisable for MIS surgeons to learn about and adopt navigation technology into our respective practices.

\section{Compliance with Ethical Standards}

Conflict of interest Jonathan N. Sembrano, MD has a research support from NuVasive, Inc. Sharon C. Yson, MD received a research support from SI-Bone, Inc. Jeffrey J. Theismann has no conflicts of interest.

Human and Animal Rights and Informed Consent No human or animal studies were conducted by authors for this manuscript.

\section{References}

Papers of particular interest, published recently, have been highlighted as:

- Of importance

•• Of major importance

1. Rampersaud YR, Foley KT, Shen AC, Williams S, Solomito M. Radiation exposure to the spine surgeon during fluoroscopically assisted pedicle screw insertion. Spine (Phila Pa 1976). 2000;25(20):2637-45.

2. Nakashima H, Sato K, Ando T, Inoh H, Nakamura H. Comparison of the percutaneous screw placement precision of isocentric C-arm 3-dimensional fluoroscopy-navigated pedicle screw implantation and conventional fluoroscopy method with minimally invasive surgery. J Spinal Disord Tech. 2009;22(7):468-72.

3. Yang BP, Wahl MM, Idler CS. Percutaneous lumbar pedicle screw placement aided by computer-assisted fluoroscopy-based navigation: perioperative results of a prospective, comparative, multicenter study. Spine (Phila Pa 1976). 2012;37(24):2055-60.

4. Bourgeois AC, Faulkner AR, Bradley YC, et al. Improved accuracy of minimally invasive transpedicular screw placement in the lumbar spine with 3-dimensional stereotactic image guidance: a comparative meta-analysis. J Spinal Disord Tech. 2015;28(9):324-9.

5. Ohba T, Ebata S, Fujita K, Sato H, Haro H. Percutaneous pedicle screw placements: accuracy and rates of cranial facet joint violation using conventional fluoroscopy compared with intraoperative three-dimensional computed tomography computer navigation. Eur Spine J. 2016;25(6):1775-80.

6. Innocenzi G, Bistazzoni S, D’Ercole M, Cardarelli G, Ricciardi F. Does navigation improve pedicle screw placement accuracy? Comparison between navigated and non-navigated percutaneous and open fixations. Acta Neurochir Suppl. 2017;124:289-95.

7. Fomekong E, Pierrard J, Raftopoulos C. Comparative cohort study of percutaneous pedicle screw implantation without versus with navigation in patients undergoing surgery for degenerative lumbar disc disease. World Neurosurg. 2018;111:e410-7.

8. Wood M, Mannion R. A comparison of CT-based navigation techniques for minimally invasive lumbar pedicle screw placement. J Spinal Disord Tech. 2011;24(1):E1-5.

9. Santos ER, Sembrano JN, Yson SC, Polly DW Jr. Comparison of open and percutaneous lumbar pedicle screw revision rate using 3D image guidance and intraoperative CT. Orthopedics. 2015;38(2): e129-34.

10. Yson SC, Sembrano JN, Sanders PC, Santos ER, Ledonio CG, Polly DW Jr. Comparison of cranial facet joint violation rates between open and percutaneous pedicle screw placement using intraoperative 3-D CT (O-arm) computer navigation. Spine (Phila Pa 1976). 2013;38(4):E251-8.
11. Tian $\mathrm{W}, \mathrm{Xu} \mathrm{Y}, \mathrm{Liu} \mathrm{B}$, et al. Lumbar spine superior-level facet joint violations: percutaneous versus open pedicle screw insertion using intraoperative 3-dimensional computer-assisted navigation. Chin Med J. 2014;127(22):3852-6.

12. von Jako R, Finn MA, Yonemura KS, et al. Minimally invasive percutaneous transpedicular screw fixation: increased accuracy and reduced radiation exposure by means of a novel electromagnetic navigation system. Acta Neurochir. 2011;153(3):589-96.

13. Villavicencio AT, Burneikiene S, Bulsara KR, Thramann JJ. Intraoperative three-dimensional fluoroscopy-based computerized tomography guidance for percutaneous kyphoplasty. Neurosurg Focus. 2005;18(3):e3.

14. Izadpanah K, Konrad G, Sudkamp NP, Oberst M. Computer navigation in balloon kyphoplasty reduces the intraoperative radiation exposure. Spine (Phila Pa 1976). 2009;34(12):1325-9.

15. Sun CT, Zhao LL, Zhang QW, Wen LY, Zhang HC. Navigation techniques assisted kyphoplasty for the treatment of osteoporotic spinal compression fracture. Chin Med J. 2009;122(8):987-9.

16. Sembrano JN, Yson SC, Polly DW Jr, Ledonio CG, Nuckley DJ, Santos ER. Comparison of nonnavigated and 3-dimensional imagebased computer navigated balloon kyphoplasty. Orthopedics. 2015;38(1):17-23.

17. Kim CW, Lee YP, Taylor W, Oygar A, Kim WK. Use of navigationassisted fluoroscopy to decrease radiation exposure during minimally invasive spine surgery. Spine J. 2008;8(4):584-90.

18. Luo W, Zhang F, Liu T, Du XL, Chen AM, Li F. Minimally invasive transforaminal lumbar interbody fusion aided with computerassisted spinal navigation system combined with electromyography monitoring. Chin Med J. 2012;125(22):3947-51.

19. Cho JY, Chan CK, Lee SH, Lee HY. The accuracy of 3D image navigation with a cutaneously fixed dynamic reference frame in minimally invasive transforaminal lumbar interbody fusion. Comput Aided Surg. 2012;17(6):300-9.

20. Zhang Y, Xu C, Zhou Y, Huang B. Minimally invasive computer navigation-assisted endoscopic transforaminal interbody fusion with bilateral decompression via a unilateral approach: initial clinical experience at one-year follow-up. World Neurosurg. 2017;106: 291-9.

21. Tian W, Xu YF, Liu B, et al. Computer-assisted minimally invasive transforaminal lumbar interbody fusion may be better than open surgery for treating degenerative lumbar disease. Clin Spine Surg. 2017;30(6):237-42.

22. Xu YF, Le XF, Tian W, et al. Computer-assisted, minimally invasive transforaminal lumbar interbody fusion: one surgeon's learning curve A STROBE-compliant article. Medicine (Baltimore). 2018;97(27):e11423.

23. Park P. Three-dimensional computed tomography-based spinal navigation in minimally invasive lateral lumbar interbody fusion: feasibility, technique, and initial results. Neurosurgery. 2015;11(Suppl 2):259-67.

24. Jiang J, Gan F, Tan H, et al. Effect of computer navigation-assisted minimally invasive direct lateral interbody fusion in the treatment of patients with lumbar tuberculosis: a retrospective study. Medicine (Baltimore). 2018;97(48):e13484.

25. Zhang YH, White I, Potts E, Mobasser JP, Chou D. Comparison perioperative factors during minimally invasive pre-psoas lateral interbody fusion of the lumbar spine using either navigation or conventional fluoroscopy. Global Spine J. 2017;7(7):657-63.

26. DiGiorgio AM, Edwards CS, Virk MS, Mummaneni PV, Chou D. Stereotactic navigation for the prepsoas oblique lateral lumbar interbody fusion: technical note and case series. Neurosurg Focus. 2017;43(2):E14.

27. Sellin JN, Mayer RR, Hoffman M, Ropper AE. Simultaneous lateral interbody fusion and pedicle screws (SLIPS) with CT-guided navigation. Clin Neurol Neurosurg. 2018;175:91-7. 
28. Gianaris TJ, Helbig GM, Horn EM. Percutaneous pedicle screw placement with computer-navigated mapping in place of Kirschner wires: clinical article. J Neurosurg Spine. 2013;19(5): 608-13.

29. Kim TT, Drazin D, Shweikeh F, Pashman R, Johnson JP. Clinical and radiographic outcomes of minimally invasive percutaneous pedicle screw placement with intraoperative CT (O-arm) image guidance navigation. Neurosurg Focus. 2014;36(3):E1.
30. Tam AL, Mohamed A, Pfister M, et al. C-arm cone beam computed tomography needle path overlay for fluoroscopic guided vertebroplasty. Spine (Phila Pa 1976). 2010;35(10):1095-9.

31. Schils F. O-arm-guided balloon kyphoplasty: prospective singlecenter case series of 54 consecutive patients. Neurosurgery. 2011;68(2 Suppl Operative):ons250-6 discussion 256.

Publisher's note Springer Nature remains neutral with regard to jurisdictional claims in published maps and institutional affiliations. 